\title{
Tratamiento microquirúrgico de los aneurismas de la bifurcación de la carótida interna
}

\author{
J.M. González-Darder; P. González-López y L. Botella-Maciá
}

Servicio de Neurocirugía. Hospital Clínico Universitario de Valencia. Servicio Valenciano de Salud.

\section{Resumen}

Se presentan los hallazgos clínicos y de imagen de una serie de 14 aneurismas de bifurcación de la arteria carótida interna tratados microquirúrgicamente. Un total de 10 lesiones no se habían roto y las $\mathbf{4}$ restantes se presentaron con hemorragia subaracnoidea y hematoma frontobasal. El diagnóstico se realizó con técnicas de neuroimagen (TAC, angio-TAC-3D; angio-RNM, angiografía), aunque los pacientes con aneurismas rotos fueron intervenidos con los hallazgos proporcionados por el angio-TAC-3D. El tamaño medio del saco era de $8.4 \mathrm{~mm}(3-13.3)$ y el del cuello $6.8 \mathrm{~mm}(3-9.6)$, con una relación saco/cuello de 1.32 (0.46-2.05). Las lesiones se trataron por vía pterional sin incidencias y con ayuda de clipaje temporal en todos los casos. Se utilizaron técnicas peroperatorias de neuroprotección, neuromonitorización y determinación de flujo vascular con micro-doppler. Los resultados clínicos han sido excelentes, con 13 pacientes con GOS 5 y uno con GOS 4 a partir de los tres meses del alta y exclusión del aneurisma en los controles angiográficos postquirúrgicos realizados en los pacientes con aneurisma roto.

PALABRAS CLAVE: Aneurisma cerebral. Hemorragia subaracnoidea. Craneotomía.

Microsurgical treatment of internal carotid bifurcation aneurysms

\section{Summary}

Clinical and imaging findings of a series of 14 internal carotid artery bifurcation aneurysms microsurgically treated are presented. A total of 10 lesions were diagnosed before rupture and 4 patients presented with subarachnoidal hemorraghe and frontobasal intracerebral bleeding. Diagnosis was done using neuroimaging (CT scan, angio-CT-3D, angio-MRI, angiography) but

Recibido: 10-03-09. Aceptado: 27-04-09 patients with ruptured aneurysms were treated with the sole information provided by the angio-CT-3D. The average fundus size was $8.4 \mathrm{~mm}(3-13.3)$ and the average neck size was $6.8 \mathrm{~mm}$ (3-9.6), being the fundus-to-neck ratio $1.32(0.46-2.05)$. All lesions were microsurgically treated through a pterional approach with the help of temporary clipping of the aferent vessesl in all cases. We used peroperative neuroprotective, neuromonitorization and micro-doppler cerebral flow measurement. Clinical results were excellent with 13 patients GOS grade 5 and one grade 4 three month afterwards of hospital discharge and complete exclusion of the lesion in angiographic controls done solely in patients with ruptured lesions.

KEY WORDS: Cerebral aneurysm. Subaracnoid hemorraghe. Craniotomy

Introducción

Los aneurismas de la bifurcación de la carótida interna (AnBIF) asientan en una zona de gran stress hemodinámico. Esta característica, junto al hecho de afectar a individuos jóvenes, tendencia al crecimiento y sangrado, alta tasa de resangrado precoz y presentar una relación cuello-fondo poco favorable, convierten a estos aneurismas cerebrales en buenos candidatos al tratamiento microquirúrgico frente al tratamiento endovascular. Sin embargo, y aun teniendo en cuenta que la incidencia de estos aneurismas sea relativamente baja, las referencias en la bibliografía sobre este subgrupo de aneurismas son muy escasas ${ }^{1,3,6,7,8,9,12,15,16}$.

El propósito de este trabajo es repasar la experiencia propia en el tratamiento microquirúrgico de los AnBIF y revisar la bibliografía al respecto para establecer las características clínicas y las pautas de manejo de los mismos.

Material y método

Se incluyen un total de 14 casos de AnBIF tratados microquirúrgicamente por el primer autor en 14 pacientes. Se han estudiado las características clínicas de los pacien- 


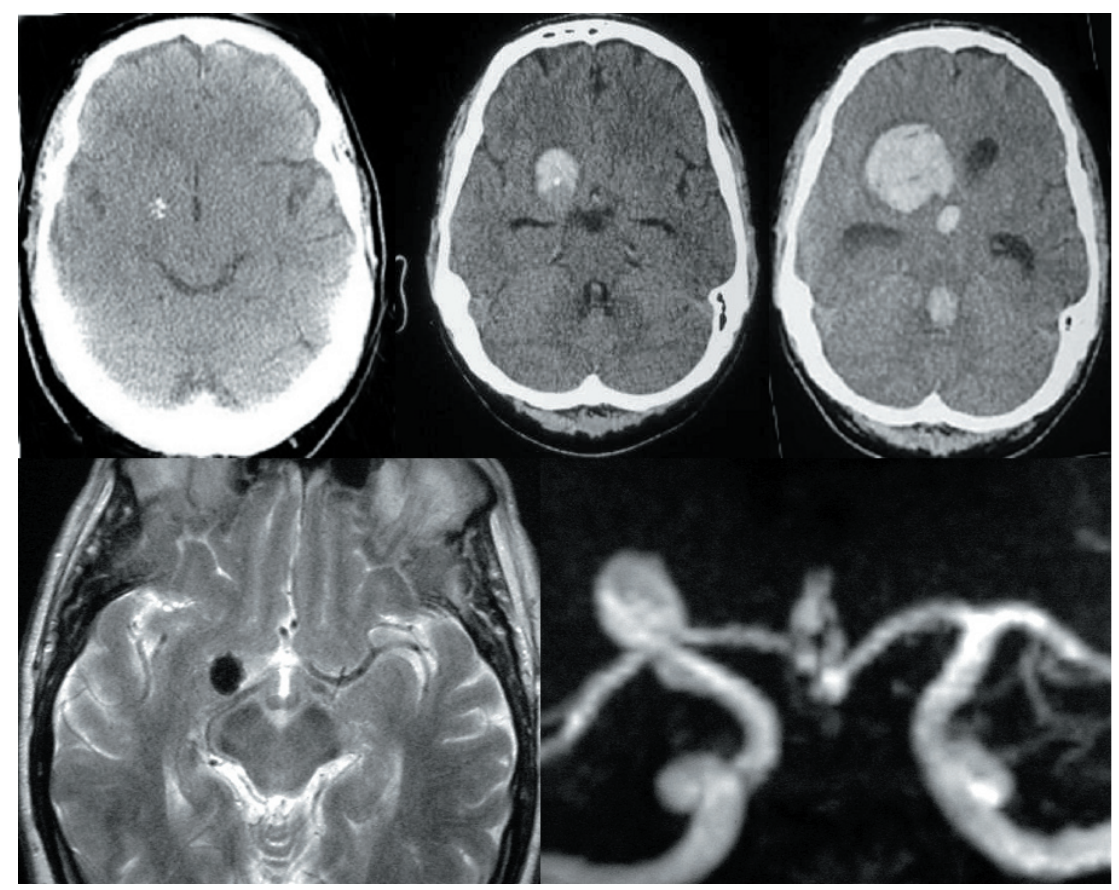

Figura 1. Diagnóstico por imagen de los AnBIF. Fila superior de izquierda a derecha: imagen de TAC con una calcificación en cisternas basales correspondiente a un aneurisma no roto; hematoma frontobasal con imagen en negativo del aneurisma roto; imagen de gran hemorragia frontobasal abierta a ventrículos. Fila inferior: estudio de RNM y angio-RNM de un aneurisma no roto.

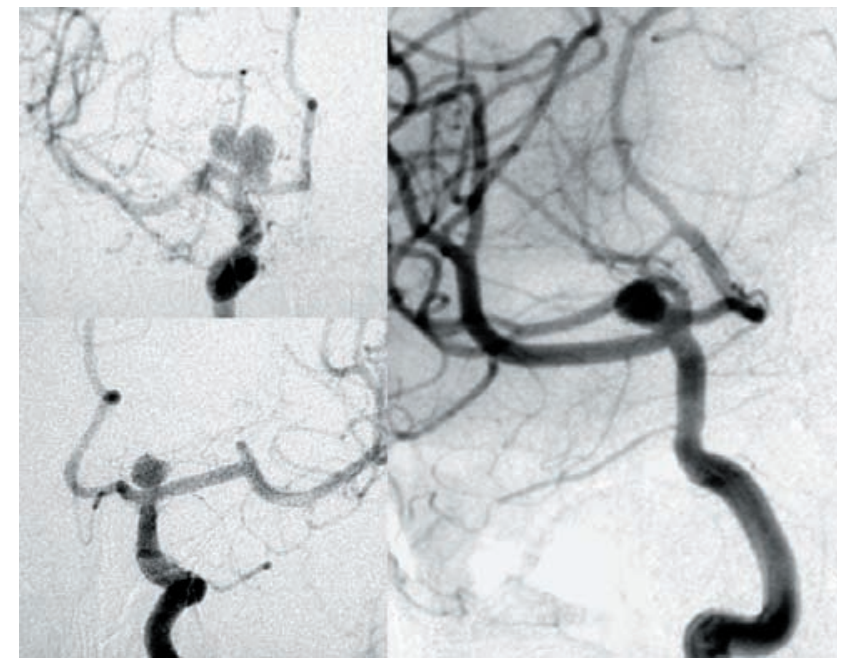

Figura 2. Diagnóstico por imagen de los AnBIF. Estudiio angiográfico anteroposterior de un aneurisma sacular multilobulado derecho (arriba-izquierda), otro simple izquierdo (abajo-izquierda) y de un aneurisma derecho, con una arteria cerebral media accesoria que sale de la misma bifurcación carotídea (derecha).

tes y de los parámetros anatómicos de las lesiones intervenidas. Para ello, los pacientes fueron clasificados a su ingreso según los grados de la World Federation of Neurological Surgeons (WFNS) ${ }^{11}$. Los pacientes que precisaban intubación orotraqueal se clasificaban como grado 4 si presentaban reactividad a los estímulos nociceptivos y como grado 5 si no presentaban reactividad o estaban en coma barbitúrico. El resultado evolutivo se ha valorado mediante

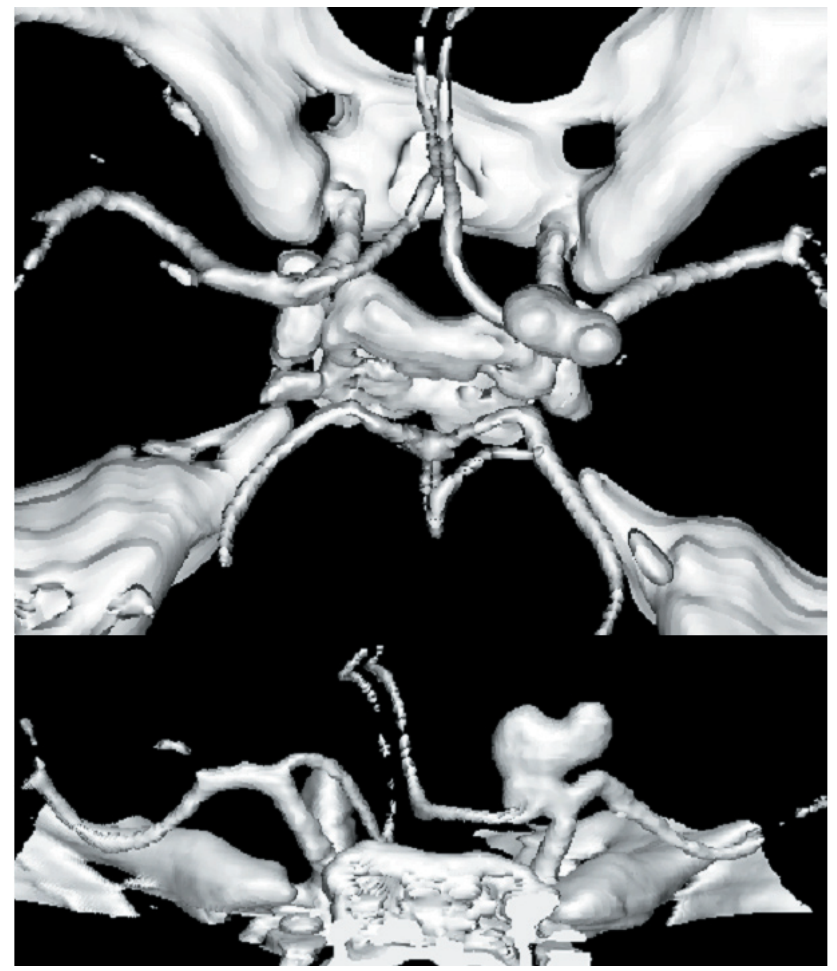

Figura 3. Diagnóstico por imagen de los AnBIF. Estudio con angio-TAC-3D de un aneurisma multilobulado derecho en una visión superior y posterior.

los grados del Glasgow Outcome Scale (GOS) ${ }^{5}$, tanto al alta hospitalaria como a los tres, seis y doce meses. Todas las incidencias del ingreso hospitalario y evolución de cada uno de los pacientes se recogían de forma sistemática en 


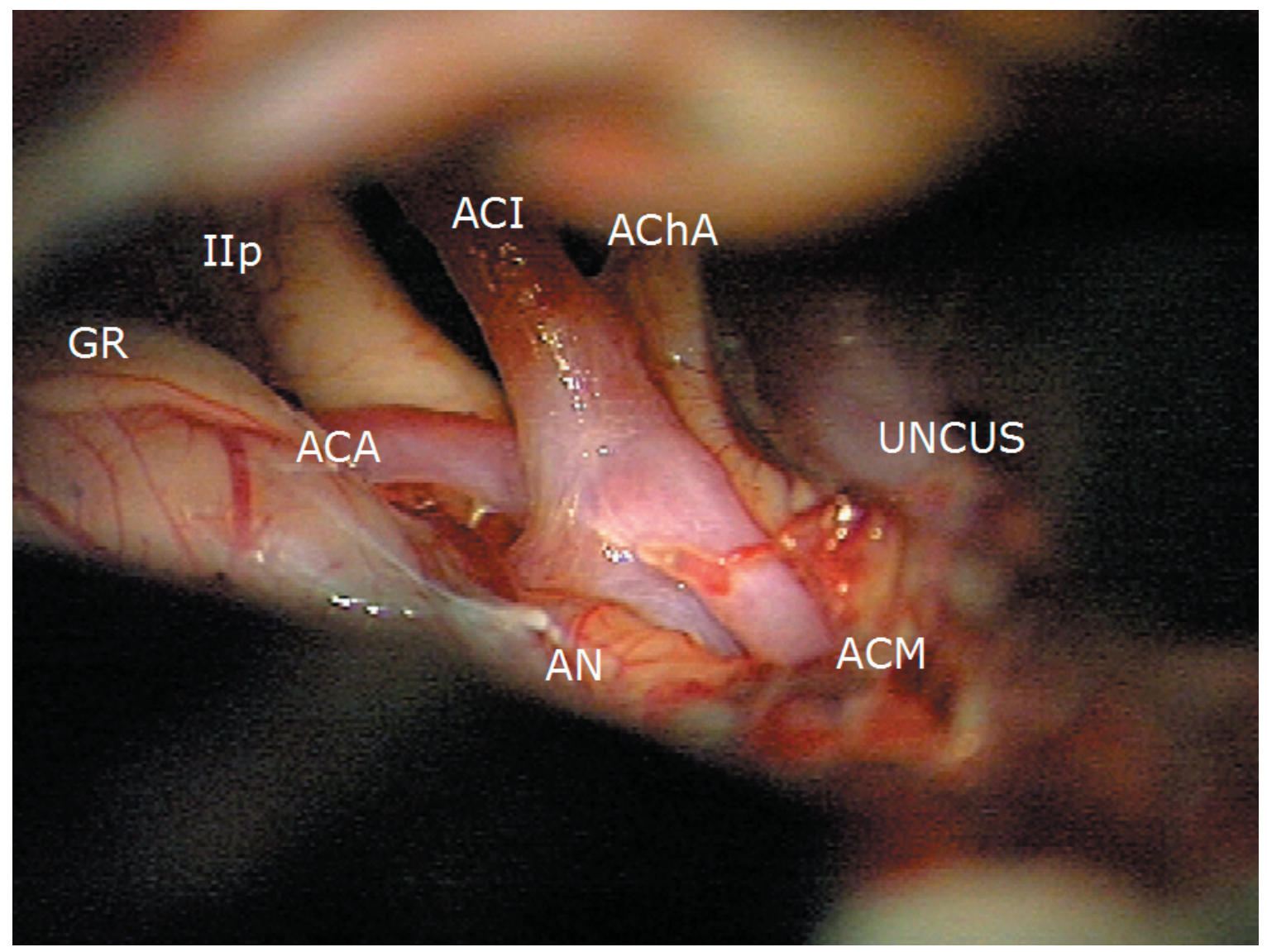

Figura 4. Imagen operatoria de un AnBIF derecho no roto, mostrando las relaciones anatómicas antes de la disección del cuello y su clipaje (IIp: nervio óptico; ACI: arteria carótida interna: AChA: arteria coroidea anterior; UNCUS: uncus del lóbulo temporal retraído por una espátula; ACM: arteria cerebral media; AN: saco aneurismática enterrado en el lóbulo frontal; ACA: segmento A1 de la arteria cerebral anterior; GR: giro recto).

una base de datos que se cumplimenta en el momento del alta hospitalaria y se actualiza en las posteriores revisiones y de donde se han extraído los datos para el presente estudio. Las características anatómicas de las lesiones se han obtenido de la revisión de los estudios de neuro-imagen y de las imágenes operatorias.

El diagnóstico y estudio preoperatorio de la lesión se ha realizado con técnicas de neuroimagen. En los casos de presentación con hemorragia subaracnoidea (HSA) se realizaba una tomografía axial computarizada (TAC) seguida de angio-TAC con reconstrucción tridimensional (angio-TAC3D), de acuerdo a un protocolo ya publicado por nosotros ${ }^{2}$. Como norma general, si los hallazgos de la angio-TAC-3D son congruentes con los datos de la exploración clínica y los hallazgos de la TAC se procede al tratamiento del paciente mediante clipaje de la lesión como primera opción terapéutica. En el resto de los pacientes y en aquellos sin HSA, el estudio de imagen podía incluir además de la angio-TAC$3 \mathrm{D}$, la imagen por resonancia nuclear magnética (RNM), angiorresonancia (angio-RNM) y la angiografía cerebral (figs. 1,2 y 3).

La cirugía se realiza a través de un abordaje pterional convencional y siguiendo los principios microquirúrgicos descritos en la literatura ${ }^{8,9,16}$. De forma rutinaria se realiza la exposición proximal de la arteria carótida interna y de la arteria cerebral anterior por encima del nervio óptico, seguida de una amplia apertura de la cisura de Silvio para identificar distalmente la arteria cerebral media, con lo que queda aislada la bifurcación carotídea (fig.4). En todo momento debe evitarse la retracción del lóbulo frontal, donde con frecuencia está enterrado el aneurisma, para evitar su ruptura. Se ha empleado sin restricciones el clipaje temporal de la carótida interna en las fases iniciales de la disección del cuello del aneurisma y el de las arterias cerebral media y anterior en las fases finales de la disección, separación de perforantes y clipaje. Como comprobación de la efectividad de la exclusión se procede sistemáticamente a la punción del saco aneurismático y, en su caso, a su exéresis. Como técnica de monitorización del flujo arterial se ha usado una sonda microdopper y, recien- 
temente, hemos introducido técnicas neurofisiológicas con registro peroperatorio de potenciales somatosensoriales y motores. El control de la cirugía se realiza mediante la angiografía cerebral postoperatoria de ambas carótidas y vertebral en proyecciones anteroposterior, lateral y ambas oblicuas.

\section{Resultados}

Los 14 casos suponen el 4.4\% de la serie de pacientes intervenidos por el primer autor. Había predominio de mujeres $(57.1 \%$ ) y la edad media era de 44.6 años (rango 17-71). En la mayor parte de los aneurismas (10 pacientes: $71.4 \%$ ) fueron diagnosticados antes de su ruptura en estudios de imagen, mientras que el resto sangraron (4 pacientes: $28,6 \%$; grados WFNS: $5,3,3,2$ ). Todos los pacientes con aneurismas rotos presentaban HSA y asociaban hematomas intraparenquimatosos frontobasales de diferente tamaño y uno de ellos tuvo un resangrado precoz. El tamaño medio de las lesiones era de $8,4 \mathrm{~mm}$ (rango 3-13.3) y el del cuello de 6.8mm (rango 3-9.6), con un índice fundus/cuello medio de 1.32 (rango 0.46-2.05). Dos lesiones eran bilobuladas y una trilobulada. La mayor parte de los aneurismas asentaban en el lado derecho (64.3\%). Todas las lesiones fueron clipadas a través de un abordaje pterional y en todas se utilizó el clipaje temporal de los vasos vecinos (media 5.4min; rango 2-13). En los pacientes con hematomas, éstos fueron evacuados. Una paciente asociaba un quiste hipofisario, que fue resecado en la misma cirugía y en otro caso se evidenció la fenestración de la arteria cerebral media pocos milímetros distalmente a la bifurcación. No hubieron complicaciones intraoperatorias. Una paciente con aneurisma no roto presentó un sangrado talámico lejano a la cirugía en el TAC de control postoperatorio, asociando una hemiparesia transitoria. Al alta el GOS de los pacientes con aneurisma no roto era de 5 y se mantuvo en los controles. A los tres meses el GOS de todos los pacientes con sangrado era de 5, excepto en una de ellas que mantuvo la hemiplegia presente al ingreso, quedando con GOS de 4. El control angiográfico se realizó sólo en los pacientes que habían sangrado, resultando en todos los casos excluido el aneurisma sin restos, recanalizaciones ni oclusión de vasos vecinos.

\section{Discusión}

En los modelos de crecimiento de los aneurismas cerebrales, las bifurcaciones arteriales son las zonas de mayor stress hemodinámico y son el lugar donde asientan de forma preferente los aneurismas, ya que son el punto donde se localizan las zonas de fallo mural con disrupción de la lámina interna. La primera bifurcación intracraneal es la de la ACI, por lo que resulta sorprendente la relativamente escasa incidencia de aneurismas en este punto. Ingebrigtsen et al. estudian el stress hemodinámico en relación a la geometría de la bifurcación y concluyen indicando que la confluencia de fuerzas hemodinámicas juegan un importante papel en la distribución del trabajo mecánico en las bifurcaciones arteriales, aunque afortunadamente la geometría de las bifurcaciones del polígono de Willis se aproxima más a los principios óptimos que las bifurcaciones distales, donde la incidencia de aneurismas es mayor ${ }^{4}$. Sakamoto et al. estudian la localización del saco de los AnBIF y demuestran que se encuentran algo desviados hacia la salida de la A1, donde el stress hemodinámico sería mayor ${ }^{12}$. Algunos casos se han descrito asociados a agenesia o fenestración de la carótida interna ${ }^{6}$, en uno de nuestros pacientes se identificó la fenestración y en otro la bifurcación precoz de la arteria cerebral media (fig.2).

Sin embargo, el aspecto anatómico mas importante para el tratamiento microquirúrgico de los AnBIF son las arterias perforantes de la zona. La bifurcación carotídea no tiene perforantes, pero una gran cantidad de ellas se dirigen a la sustancia perforada anterior que se encuentra en íntima relación con la misma. El origen de estas perforantes es múltiple: arteria comunicante posterior, arteria coroidea, segmento A1 de la arteria cerebral anterior, segmento M1 de la arteria cerebral media en forma de las primeras lenticuloestriadas y arteria recurrente de Heubner, procedente de la unión A1A2 de la cerebral anterior ${ }^{8,9,12}$. La mayor parte de estos vasos son invisibles en la angiografía cerebral, excepción hecha de la recurrente, cuyas relaciones con el aneurisma pueden ser determinadas preoperatoriamente.

La incidencia de los AnBIF recogida en la literatura se encuentra entre el 2.4 y el $8.9 \% \%^{1,3,8,9,16}$, mientras que la de nuestra serie es del $4.4 \%$ de los aneurismas rotos y no rotos intervenidos. La incidencia variable en las diferentes series se relaciona con la posible inclusión o no en esta localización de lesiones distales de la ACI o proximales de la cerebral media o anterior. La presentación clínica mas frecuente es la hemorragia subaracnoidea, aunque en nuestra serie la mayor parte de los casos intervenidos fueron descubiertos de forma casual. En la literatura se describe que algunos pacientes se presentan con clínica oftalmológica por compresión de la cintilla óptica. Dado que el aneurisma se encuentra enterrado en el seno del lóbulo frontal, es típica la asociación de un hematoma frontal que asciende hacia el caudado y puede abrirse al ventrículo lateral, asociando con frecuencia déficit de vías largas. Como sucede en las series descritas, la mayor parte de nuestros pacientes eran de sexo femenino y muchos de ellos jóvenes. Este último hecho supone la necesidad de estudiar factores de riesgo asociados, especialmente displasias vasculares, conectivopatías y poliquistosis renal. En el momento del diagnóstico, los aneurismas suelen ser de tamaño medio o grande, siendo excepcionales los aneurismas gigantes. El tamaño medio 
de los AnBIF de nuestra serie es de $8.4 \mathrm{~mm}$, con un cuello relativamente ancho de $6.8 \mathrm{~mm}$ de media y muchos de ellos con varias lobulaciones. Dado que el aneurisma se encuentra lejos de estructuras óseas, el diagnóstico con técnicas de imagen como RNM y TAC es relativamente fácil (fig.1). En nuestra experiencia, muchos de los pacientes fueron intervenidos con la información proporcionada únicamente con el angio-TAC-3D (fig.3) y en el resto se asoció la angioRNM y la angiografía. En todos los pacientes con sangrado se asociaba un hematoma frontobasal, abierto a ventrículo, muy sugestivo de aneurisma de esta localización y que en un caso se asociaba a una hemiplegia contralateral que se mantuvo al final del seguimiento.

En nuestra serie, la relación fundus/cuello (1.32) era muy desfavorable para el tratamiento endovascular. Otros factores desfavorables son la localización del cuello enfrentado al flujo, la edad de los pacientes, la tendencia reconocida en la literatura para el sangrado y el resangrado precoz y la frecuente asociación de un hematoma intraparenquimatoso. Finalmente, en las series de tratamiento endovascular se publican altos índices de recanalización y de retratamiento ${ }^{1,10,15}$. Todo ello hace que el tratamiento microquirúrgico sea el de elección. Algunas publicaciones describen los aspectos generales del abordaje quirúrgico de los $\mathrm{AnBIF}^{8,9,16}$. En nuestra experiencia, los AnBIF requieren del uso de la mayor parte de las estrategias quirúrgicas y de monitorización desarrolladas para el manejo microquirúrgico de los aneurismas cerebrales. Se requiere el abordaje inicial a la ACI para control proximal y drenaje de las cisternas; después se aborda la cisura de Silvio hasta exponer el segmento M1 de la cerebral media y primera lenticuloestriada; se vuelve ahora a la ACI para llegar a la cerebral anterior entre el quiasma y el lóbulo frontal, con una mínima retracción del mismo; y, finalmente, se abre la porción mas proximal y profunda de la cisura de Silvio con lo que se expone la bifurcación por su parte inferior y así el cuello del aneurisma (fig.4). Tras introducir las técnicas anestesiológicas de neuroprotección y activar las técnicas de neuromonitorización somatosensorial y de la vía motora, se coloca un clip temporal en la ACI distalmente a la coroidea anterior y se inicia la disección del cuello y de las perforantes de la zona. En las fases finales de la disección y para el clipaje pueden colocarse clips temporales adicionales en la cerebral media y/o anterior. Tras el clipaje del cuello se retiran los clips temporales y se reconoce el flujo de los vasos de gran calibre con una microsonda doppler. En la actualidad, es posible evidenciar la permeabilidad de las perforantes con la microangiografía con fluorescencia ${ }^{14}$. Se explora cuidadosamente la zona para recolocar el clip en caso necesario. Puede ahora exponerse el saco aneurismático separándolo de la base del lóbulo frontal, vaciarlo o extirparlo y, finalmente, evacuar el hematoma intracerebral que pudiera existir. Antes de cerrar se aplica un vasodilatador sobre los vasos y, en caso de hemorragia intraventricular, se abre la lámina terminalis.

En nuestra experiencia, los resultados quirúrgicos y clínicos son muy buenos. Sólo una paciente con hemiplegia al ingreso mantuvo dicho déficit neurológico al alta. Otra paciente presentó un sangrado espontáneo a distancia, probablemente secundario a una angiopatía, y cursó con una leve hemiparesia transitoria. El resto de los pacientes mostraron una evolución sin incidencias reseñables. Todos los aneurismas fueron excluidos sin restos aneurismáticos. Los datos de la literatura son semejantes, con muy baja morbimortalidad $^{1,3,8,9,12,15,16}$.

En conclusión, los AnBIF constituyen un subgrupo de aneurismas cerebrales con características propias y con buenos resultados cuando son tratados con técnicas microquirúrgicas.

\section{Agradecimientos}

El primer autor quiere agradecer a todo el personal que ha colaborado en el diagnóstico y tratamiento de los pacientes incluidos en el estudio, en especial a los miembros de los Servicios de Neurocirugía del Hospital General de Castellón y del Hospital Clínico Universitario de Valencia.

\section{Bibliografía}

1. Geyik, S., Yavuz, K., Cekirge, S., Saatci, I.: Endovascular treatment of basilar and ICA termination aneurysms: effects of the use of hydroCoils on treatment stability in a subgroup of patients prone to a higher recurrence rate. Neuroradiology 2007; 49: 1015-21.

2. González-Darder, J.M., Pesudo-Martínez, J.V., FeliuTatay, R.A.: Microsurgical management of cerebral aneurysms based in CT angiography with three-dimensional reconstruction (3D-CTA) and without preoperative cerebral angiography. Acta Neurochir. (Wien) 2001; 143: 673-679.

3. Gupta, S.K., Khosla, V.K., Chhabra, R., Mohindra, S., Bapuraj, J.R., Khandelwal, N., Mukherjee, K.K., Tewari, M.K., Pathak, A., Mathuriya, S.N.: Internal carotid artery bifurcation aneurysms: surgical experience. Neurol Med Chir (Tokyo) 2007; 47: 153-157.

4. Ingebrigtsen, T., Morgan, M.K., Faulder, K., Ingebrigtsen, L., Sparr, T., Schirmer, H.: Bifurcation geometry and the presence of cerebral artery aneurysms. J Neurosurg 2004; 101: 108-113.

5. Jennet, B., Bond, M.: Assessment of outcome after severe brain damage. A practical scale. Lancet 1975; 1: 480-484.

6. Hattori. T., Kobayashi, H.: Fenestration of the supraclinoid internal carotid artery associated with carotid bifurcation aneurysm. Surg Neurol 1992; 37: 284-288. 
7. Miyazawa, N., Nukui, H., Horikoshi, T., Yagishita, T., Sugita, M., Kanemaru, K.: Surgical management of aneurysms of the bifurcation of the internal carotid artery. Clin Neurol Neurosurg 2002; 104: 103-14.

8. Osawa, M., Kobayashi, S., Tanaka, Y.: Internal carotid bifurcation aneurysms: Surgical techniques. En Batjer, H.H. (ed). Cerebrovascular Disease, Lippincott-Raven, Philadelphia, 1997; 983-992.

9. Ogilvy, C.S., Crowell, R.M.: Carotid bifurcation aneurysms. En Ojemann RG, Ogilvy CS, Crowell RM y Heros RC (eds). Surgical Management of Neurovascular Disease ( $3^{\mathrm{a} e d}$.), Williams\&Wilkins, Baltimore, 1995; 227-237.

10. Raftopoulos, C., Goffette, P., Vaz, G., Ramzi, N., Wittebole, X., Mathurin, P.: Surgical clipping may lead to better results than coil embolization :results from a series of 101 consecuive unruptured intracranial aneurysms. Neurosurgery 2003; 52: 1280-1290.

11. Report of the World Federation of Neurological Surgeons Committee on a Universal SAH Grading Scale.: J. Neurosurg. 1998; 68: 985-986.

12. Rhoton, A.L.: The supratentorial arteries. Neurosurgery 2002: 51 (Suppl 1): 53-120.

13. Sakamoto, S., Ohba, S., Shibukawa, M., Kiura, Y.,

\begin{abstract}
Comentario al trabajo: Tratamiento microquirúrgico de los aneurismas de la bifurcación de la carótida interna de J.M. González-Darder y cols
\end{abstract}

González-Darder et al nos demuestran que la cirugía de los aneurismas de la bifurcación carotídea tiene unos resultados mejores que con la embolización, que no es necesario un centro de tercer nivel para obtenerlos, si existe un equipo multidisciplinario motivado y que los aneurismas de esta localización tienen unas peculiaridades especiales. Nosotros tenemos una incidencia similar a la de los autores aunque el porcentaje de aneurismas "no rotos" es inferior. La cuestión no es cirugía versus embolización sino cirugía y embolización. Muchos estamos convencidos de que se hará embolización y/o cirugía por el mismo especialista en un quirófano dotado para ambos procedimientos. Muchas veces operando de urgencias a un paciente con una hemorragia subaracnoidea aneurismática he pensado viendo el cerebro "rojo, hinchado y enfadado" que si hubiese podido
Okazaki, T., Arita, K., Kurisu, K.: Characteristics of aneurysms of the internal carotid artery bifurcation. Acta Neurochir (Wien) 2006; 148: 139-143.

14. Suzuki, K., Kodama, N., Sasaki, T., Matsumoto, M., Ichikawa, T., Munakata, R., Muramatsu, H., Kasuya, H.: Confirmation of blood flow in perforating arteries using fluorescein cerebral angiography during aneurysm surgery. $\mathrm{J}$ Neurosurg 2007: 107: 68-73.

15. van Rooij, W.J., Sluzewski, M., Beute, G.N.: Internal carotid bifurcation aneurysms: frequency, angiographic anatomy and results of coiling in 50 aneurysms. Neuroradiology 2008; 50: 583-587.

16. Yasargil, M.G.: Microneurosurgery, vol 1, New York; Thieme-Stratton, 1984.

González-Darder, J.M.; González-López, P.; BotellaMaciá, L.: Tratamiento microquirúrgico de los aneurismas de la bifurcación de la carótida interna. Neurocirogía 2010; 21: 205-210.

Correspondencia postal: Dr. José M González-Darder. Servicio de Neurocirugía. Hospital Clínico Universitario. Avda. Blasco Ibáñez, 17. 46010 Valencia embolizar antes el aneurisma, la evacuación del hematoma hubiese sido más sencilla y menos peligrosa.

La angio-TC proporciona una información similar a la angiografía con sustracción digital y en algunos centros es la única prueba que se utiliza para el tratamiento quirúrgico de muchos aneurismas, el estudio de González-Darder et al así lo corrobora.

Los avances tecnológicos no son exclusivos de las técnicas endovasculares, González-Darder et al refieren que la utilización de potenciales evocados intraoperatorios y microdoppler junto a la monitorización de la ptiO2 y a la microangiografía con fluorescencia hacen que la cirugía sea, cada vez, mas segura y efectiva.

J. Vilalta Barcelona 